\title{
THE CHARACTER OF EMBEDDED RINGS
}

\author{
TAPAN K. CHATTERJEE \\ Facultad de Ciencias, Fisico-Matematicas, \\ Universidad A. Puebla, A.P. 1316, Puebla, Mexico
}

It is well known that, under favorable conditions, tidal interactions between a spiral galaxy and a more compact elliptical leads to the formation of embedded rings in the disk (e.g. Chatterjee 1979, Bull. Astron. Soc. India, $7,32)$; in addition there are also nuclear rings whcih do not seem to have a tidal origin (e.g. Buta 1986, ApJ, 306, 768). The two types of rings occur in different regions of the spirals, which can be explained on the basis of the tidal hypothesis by extending the previous research work of Chatterjee (1984, Ap\&SSS, 106, 309). In this context, using the same theory, we study many normal on-axis collisions (as rings are best formed for this orientation) between a disk galaxy, modeled as an exponential disk with a polytropic $n=0,3,4$, bulge (mass being equally distributed amongst the polytropic indices), and a compact spherical intruder, modeled with a polytropic $n=2,3,4$, distribution (mass being equally distributed amongst the polytropic indices). The internal energy changes suffered by the disk, have a direct bearing on the sharpness of the rings, and are directly obtained from the relationship, for the fractional change in internal energy of the disk, as $f_{\mathrm{E}}=\gamma \beta$, where $\beta$ is a function of the galactic models and the collision parameter $\gamma=\mathrm{G}\left(\sigma_{\mathrm{s}} / V^{2}\right) \propto \sigma_{\mathrm{s}} / V^{2}$ (where $\sigma_{\mathrm{s}}$ is the projected velocity of collision). We find that sharp rings form for $f_{\mathrm{E}} \geq 0.5$; beyond this value of $f_{\mathrm{E}}$ the intensity contrast of the rings diminish. However, fairly sharp rings (from an observational point of view) form for $f_{\mathrm{E}} \geq 1 / 3$, which corresponds to $\gamma \geq 0.01$; which corresponds to a density maximum near a region $\sim(1 / 3) R$ ( $R$ being the radius of the disk galaxy), so that rings of tidal origin are not expected to be prominent at a distance interior to $\sim 1 / 3$ of the radius of the disk galaxy. They are not expected to form interior to $\sim 1 / 10$ of the radius of the disk. 